ACCEPTED FOR PUBLICATION IN APJ, 2009 JULY 20 v700 ISSUE

Preprint typeset using LTEX style emulateapj v. 04/20/08

\title{
DETECTION OF SUPERSONIC HORIZONTAL FLOWS IN THE SOLAR GRANULATION
}

\author{
L.R. BELLOT RUBio \\ Instituto de Astrofísica de Andalucía (CSIC), Apdo. de Correos 3004, 18080 Granada, Spain \\ Accepted for publication in ApJ, 2009 July 20 v700 issue
}

\begin{abstract}
Hydrodynamic simulations of granular convection predict the existence of supersonic flows covering $~ 3-4 \%$ of the solar surface at any time, but these flows have not been detected unambigously as yet. Using data from the spectropolarimeter aboard the Hinode satellite, I present direct evidence of fast horizontal plasma motions in quiet Sun granules. Their visibility increases toward the limb due to more favorable viewing conditions. At the resolution of Hinode, the horizontal flows give rise to asymmetric intensity profiles with very inclined blue wings and even line satellites located blueward of the main absorption feature. Doppler shifts of up to $9 \mathrm{~km} \mathrm{~s}^{-1}$ are observed at the edges of bright granules, demonstrating that the flows reach supersonic speeds. The strongest velocities occur in patches of $0.5^{\prime \prime}$ or less. They tend to be associated with enhanced continuum intensities, line widths, and equivalent widths, but large values of these parameters do not necessarily imply the existence of supersonic flows. Time series of spectropolarimetric measurements in regions away from disk center show the transient nature of the strong horizontal motions, which last only for a fraction of the granule lifetime. Supersonic flows are expected to produce shocks at the boundaries between granules and intergranular lanes, and may also play a role in the emergence of small-scale magnetic fields in quiet Sun internetwork regions.
\end{abstract}

Subject headings: Sun: granulation - Sun: photosphere - polarization

\section{INTRODUCTION}

Granules and intergranular lanes are the manifestation of overshooting convection in the solar photosphere, a process that has been studied in detail by means of 2D and 3D simulations (e.g., Stein \& Nordlund 1989; Cattaneo et al. 1990; Steffen \& Freytag 1991; Stein \& Nordlund 1998; Steiner et al. 1998; Gadun et al. 1999; Ploner et al. 1999). One of the most striking results of the simulations is the existence of supersonic horizontal flows in granules. As explained by Stein $\&$ Nordlund (1998), these flows are a natural consequence of mass conservation in a highly stratified atmosphere. The vertical upflows of granules turn into horizontal flows relatively soon due to the exponential decrease of the density with height. All the mass emerging into the photosphere through the granular cross section must leave the granule through its edges. Since the lateral area available for the outflow is much smaller than the cross section of the granule, the horizontal flow is forced to accelerate to conserve mass. The larger the granule, the more pronounced the area difference and the stronger the horizontal flow. Occasionally, supersonic velocities are reached.

The detection of these motions is challenging for a number of reasons. First of all, they require spectroscopic measurements near the limb, in order to maximize the projection of the horizontal velocity to the line of sight. Also, high angular resolution is needed to identify the spectral signatures of strong flows, which may disappear if atmospheres with different properties are mixed in the resolution element. Unfortunately, the spatial resolution is greatly reduced towards the limb because of geometrical foreshortening and because of the lower intensity contrast, which decreases the performance of adaptive optics systems.

Given these difficulties, the efforts have concentrated on the detection of shocks rather than the supersonic flows themselves. According to the simulations, shocks can be produced

Electronic address: lbellot@iaa.es by the deceleration of transonic flows in intergranular lanes or by the collision of fast flows from adjacent granules (Cattaneo et al. 1990; Stein \& Nordlund 1998), with signatures that are visible on larger scales. This means that the requirement of extremely high spatial resolution can be relaxed to some degree. In addition, shock observations are possible closer to the disk center (because the detection does not rely on Doppler shift measurements).

The first indications of shocks were obtained by Nesis et al. (1992), who observed enhanced line widths in intergranular lanes and interpreted them as increased turbulence due to the passage of shock fronts. Solanki et al. (1996) also detected broad intensity profiles compatible with those expected from shocks. More recently, Rybák et al. (2004) have identified clear shock signatures in spectroscopic observations of a granular region taken with adaptive optics. For the first time, the temporal evolution of a shock event could be followed and compared with predictions from numerical simulations.

While the existence of shocks can be considered well established by now, the motions causing them have not been observed yet. The purpose of this paper is to provide unequivocal evidence of supersonic horizontal flows in solar granular convection. They are detected as strongly Doppler shifted satellites in the Fe I $630.2 \mathrm{~nm}$ lines recorded by Hinode away from disk center. Their signatures and basic properties are studied in $\S 3$. $\S 4$ and $\S 5$ deal with the spatial distribution and temporal evolution of the flows. Finally, $\$ 6$ summarizes the main results of this work.

\section{OBSERVATIONS AND DATA ANALYSIS}

The observations analyzed here were taken with the spectropolarimeter (SP; Lites et al. 2001) of the Solar Optical Telescope (Tsuneta et al. 2008) aboard the Hinode satellite (Kosugi et al. 2007). This instrument measures the polarization spectra of the Fe I lines at 630.15 and $630.25 \mathrm{~nm}$. The wavelength sampling is $2.153 \mathrm{pm} \mathrm{pixel}^{-1}$. With a slit width and a pixel size of $0.16^{\prime \prime}$, the Hinode/SP achieves a spatial 

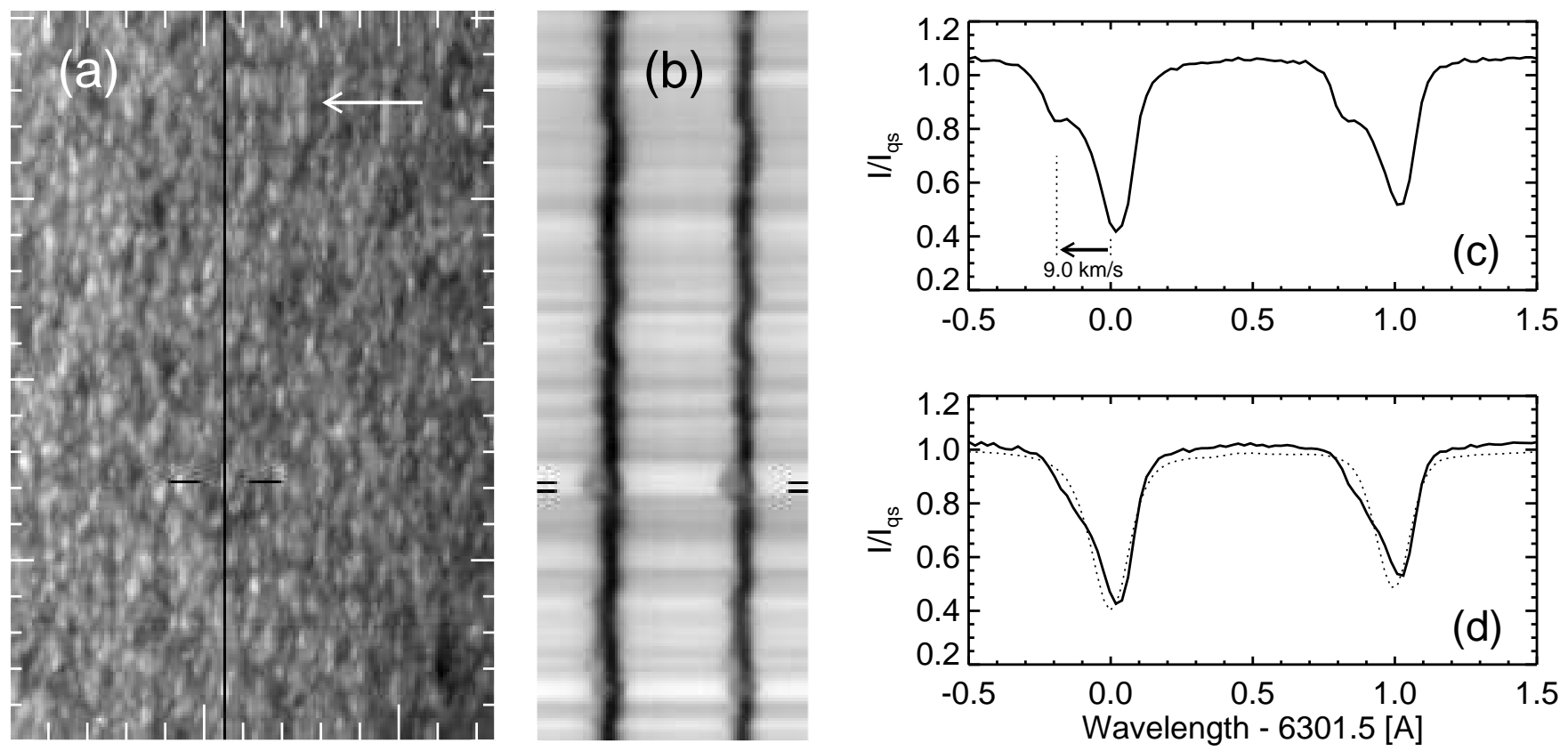

FIG. 1._- (a) Quiet Sun region observed with the Hinode/SP on September 9, 2007. The figure displays a field of view (FOV) of $24.8^{\prime \prime} \times 40.5^{\prime \prime}$, with North at the top and West to the right. Tickmarks are separated by $2^{\prime \prime}$. The heliocentric distance varies between $70^{\circ}$ and $75^{\circ}(\mu=0.34$ and 0.26$)$ from left to right. The arrow indicates the direction to disk center. The vertical line marks a slit position that crosses several granules, one of which is identified with horizontal dashes. (b) Intensity profiles of the Fe I 630.15 and $630.25 \mathrm{~nm}$ lines along the slit displayed in panel $a$. Wavelength increases from left to right, covering an interval of $0.2 \mathrm{~nm}$. Two spatial positions are selected for further analysis (short horizontal dashes). The distance between them is $0.48^{\prime \prime}$. (c) Intensity profiles emerging from the upper spatial position indicated in panel $b$. The zero of the wavelength scale corresponds to the line core position of the average Fe I $630.15 \mathrm{~nm}$ profile in the quiet Sun. (d) Same, for the other spatial position marked in panel $b$. The dotted line represents the average quiet-Sun profile.

resolution of about $0.32^{\prime \prime}$.

The data have been corrected for dark current, flatfield, and various instrumental effects using the sp_prep.pro routine included in the SolarSoft package. Three quiet Sun regions observed on 2007 September 9, 15, and 24 at heliocentric angles between $40^{\circ}$ and $75^{\circ}$ will be considered in this paper. The measurements were taken in the framework of the Hinode Operation Plans 14 and 25, both of which involved joint campaigns with the four solar telescopes of the Canary Islands (Spain). The observed Fe I $630.15 \mathrm{~nm}$ profiles have been used to compute continuum intensities, residual intensities, equivalent widths, full widths at half maximum, linecore velocities, and line bisectors at different intensity levels. In addition, the total polarization has been determined as $\mathrm{TP}=\int\left(Q^{2}+U^{2}+V^{2}\right)^{1 / 2} / I_{\mathrm{qs}} \mathrm{d} \lambda$, where $I_{\mathrm{qs}}$ is the quiet Sun continuum at $630.2 \mathrm{~nm}$. The integral extends over a wavelength range of $0.1098 \mathrm{~nm}$ to encompass the Fe I $630.15 \mathrm{~nm}$ line.

\section{SIGNATURES OF SUPERSONIC HORIZONTAL FLOWS}

At high spatial resolution, supersonic granular flows leave distinct spectroscopic signatures in the form of line satellites and very asymmetric intensity profiles. Examples of such fingerprints are given in this Section.

Figure $1 a$ shows the continuum intensity map of a quiet Sun region observed on September 9, 2007 near the West limb. The heliocentric distance varies between $70^{\circ}$ and $75^{\circ}$ from left to right. The granules appear as elongated structures due to the strong foreshortening in the E-W direction, but otherwise they are clearly visible. Figure $1 b$ displays the intensity profiles of the two Fe I lines along the slit indicated in panel $a$. The slit crosses several granules and intergranular lanes. Because of the large heliocentric angles, the typical Doppler shifts associated with granulation at disk center are no longer seen. Indeed, the spectra show small wavelength displacements except for the bright granule marked with horizontal dashes. The intensity profiles emerging from this granule are remarkable because of their very extended blue wings. The absorption in the wing is so large that it produces a line satellite. Similar features have been reported before, but only in magnetic structures harboring very strong flows as, for example, sunspot penumbrae (e.g., Bumba 1960; Wiehr 1995; Bellot Rubio 2009). Interestingly, no satellites are observed to the red anywhere along the slit.

Figures $1 c$ and $1 d$ show intensity profiles from two spatial positions within the granule. One of them (panel $c$ ) is at the edge of the granule facing the observer, roughly on the line connecting it with the disk center (hereafter referred to as the line of symmetry). The other position (panel $d$ ) also samples the outer parts of the granule, but more perpendicularly to the line of symmetry. The profiles displayed in Figure $1 c$ exhibit conspicuous line satellites in the blue wing. The satellite of Fe I $630.15 \mathrm{~nm}$ has an inflection point that seems to mark the position of its core. It is located at $\Delta \lambda=-19 \mathrm{pm}$ from the center of the average quiet Sun profile at the same heliocentric angle. This corresponds to a line-of-sight (LOS) velocity of $9.0 \mathrm{~km} \mathrm{~s}^{-1}$ towards the observer. Note that the actual flow speed must be larger due to projection effects.

In principle, absorption at such large distances in the line wing can also be produced by slower flows, but they would have to be associated with microturbulent velocities of $\sim$ $4 \mathrm{~km} \mathrm{~s}^{-1}$. Those values are unrealistically large. Even if they exist, however, LOS velocities of $\sim 4-5 \mathrm{~km} \mathrm{~s}^{-1}$ would still be required to explain the substantial wavelength shift of the satellites.

The velocities derived from the Fe I $630.2 \mathrm{~nm}$ lines refer 

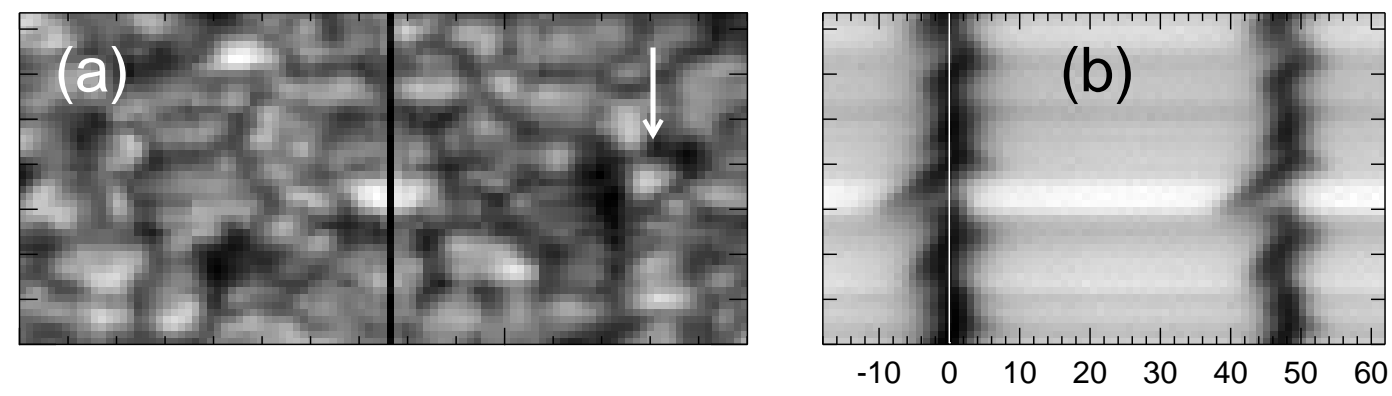

FIG. 2.- (a) $15.0^{\prime \prime} \times 7.4^{\prime \prime}$ region scanned with the Hinode/SP on September 24, 2007 at $\theta=60^{\circ}$. The arrow indicates the direction to disk center. Tickmarks are separated by $1^{\prime \prime}$. (b) Spectrogram along the slit marked in panel $a$. The wavelength axis is given in $\mathrm{km} \mathrm{s}^{-1}$. The zero velocity (vertical line) corresponds to the average line-core position of Fe I $630.15 \mathrm{~nm}$ in the displayed FOV.

to the optical depth range from $\log \tau_{5} \sim 0$ to -2 (Cabrera Solana et al. 2005). Using the temperatures, densities, and gas pressures of the Harvard-Smithsonian Reference Atmosphere (Gingerich et al. 1971), the sound speed can be estimated to be 7.7 and $7.0 \mathrm{~km} \mathrm{~s}^{-1}$ where the optical depth reaches those values at a heliocentric angle of $70^{\circ}$. The local sound speed may be even lower in the cooler granular regions. Thus, the wavelength shifts of $\sim 9 \mathrm{~km} \mathrm{~s}^{-1}$ observed in Figure $1 c$ imply supersonic flows, with Mach numbers of at least 1.2.

By contrast, the profiles displayed in Figure $1 d$ do not show obvious line satellites. They exist, but cannot be clearly identified because they are blended with the main absorption feature as a consequence of smaller Doppler shifts. Nevertheless, the flows still produce strongly distorted profiles with extended blue wings, similar to those observed by Solanki et al. (1996). Solanki et al. (1996) mentioned two different mechanisms capable of producing such broad profiles: supersonic granular flows, and the shocks associated with them. In the absence of line satellites they could not favor the first scenario, but at the resolution of Hinode it seems clear that the distorted profiles are caused by supersonic motions.

Furthermore, the flows producing the satellites and the extended blue wings have to be essentially radial. This would explain why, in the external parts of the granule facing the disk center, the wavelength shift of the satellite is maximum on the line of symmetry and very small perpendicularly to it. The transition between the two situations is smooth, as demonstrated by Figure $1 b$.

Inspection of Figures $1 c$ and $1 d$ reveals that the cores of the main absorption features are slightly redshifted. The maximum redshifts occur at the granular edges (where the satellites show stronger blueshifts), and tend to disappear toward the center of the granule. The main absorption feature is likely to get a significant contribution from (or even be entirely produced by) the intergranular plasma crossed by the slanted lines of sight, which cut the adjacent intergranular lane before reaching the granule. It is also possible that granular and intergranular profiles get mixed at the border of the granule due to the increasingly larger surface area covered by the pixel far from disk center. Both scenarios are consistent with the fact that the line-core redshifts detected at the very edge of the granule and the intergranular lane next to it are essentially the same.

The radial variation of the satellites across granules is examined in Figure 2. The displayed region was located at a heliocentric angle of $60^{\circ}$ towards the North solar pole. The vertical line in panel $a$ indicates a slit position intersecting a granular cell through its center. The lower part of the granule faces the observer, while the upper half is closer to the limb. Figure $2 b$ depicts the intensity profiles recorded along the slit. Clearly, the line satellite shows maximum blueshifts at the border of the granule in the direction to disk center. The blueshift decreases monotonically as the limbward edge of the granule is approached. At that position the satellite has merged with the main absorption feature and is no longer distinguishable. Beyond the granule, the slit crosses a narrow intergranular lane and small redshifts are detected. This behavior is typical and can also be observed in the examples of Figure 5.

The variation of the Doppler shift described above is consistent with a radial outflow that is vertical at the center of the granule and more horizontal near the edges. It is likely that the absence of redshifted satellites on the far side of the granules is due to the flow occurring below the $\tau=1$ level there. The distance that the line of sight must travel through the opaque granular plasma before reaching the horizontal flow is larger in that part of the granule. This means that the medium could become optically thick well before the flow is reached; in that case, the flow would be unable to produce any redshift in the emergent intensity profiles. While the existence of an opacity effect is clear from the observations, its origin is still uncertain. A detailed analysis of hydrodynamic simulations may help understand why the opacity is increased on the far side of the granules, leading to a complete obscuration of the flow.

As expected for horizontal motions, the line satellites are visible at relatively large heliocentric angles and not close to disk center. Their increased visibility towards the limb results from the progressively larger LOS component of the flow, which induces a stronger Doppler shift. Very close to the limb, however, the satellites disappear due to (a) the large surface area covered by the pixel, which decreases the filling factor of the horizontal flow and mixes granular and intergranular regions, and (b) the progressively higher formation height of the line, which eventually leaves the layers where overshooting convection occurs. In the future, these effects should be verified using hydrodynamic simulations.

\section{SPATIAL DISTRIBUTION}

Fast horizontal flows can be identified in the Hinode/SP maps through a line bisector analysis. The most blueshifted satellites are usually weak, therefore it is important to use bisectors at high intensity levels in order to pick out those cases too. After experimenting with different intensity levels and velocity thresholds, a good proxy for fast (supersonic) flows is to have bisector velocities of at least $-2.6 \mathrm{~km} \mathrm{~s}^{-1}$ at the $70 \%$ intensity level. For comparison, the bisector shifts of the $630.15 \mathrm{~nm}$ profiles displayed in Figures $1 c$ and $1 d$ are -2.6 and $-1.3 \mathrm{~km} \mathrm{~s}^{-1}$, respectively. That is, the first pixel would be 


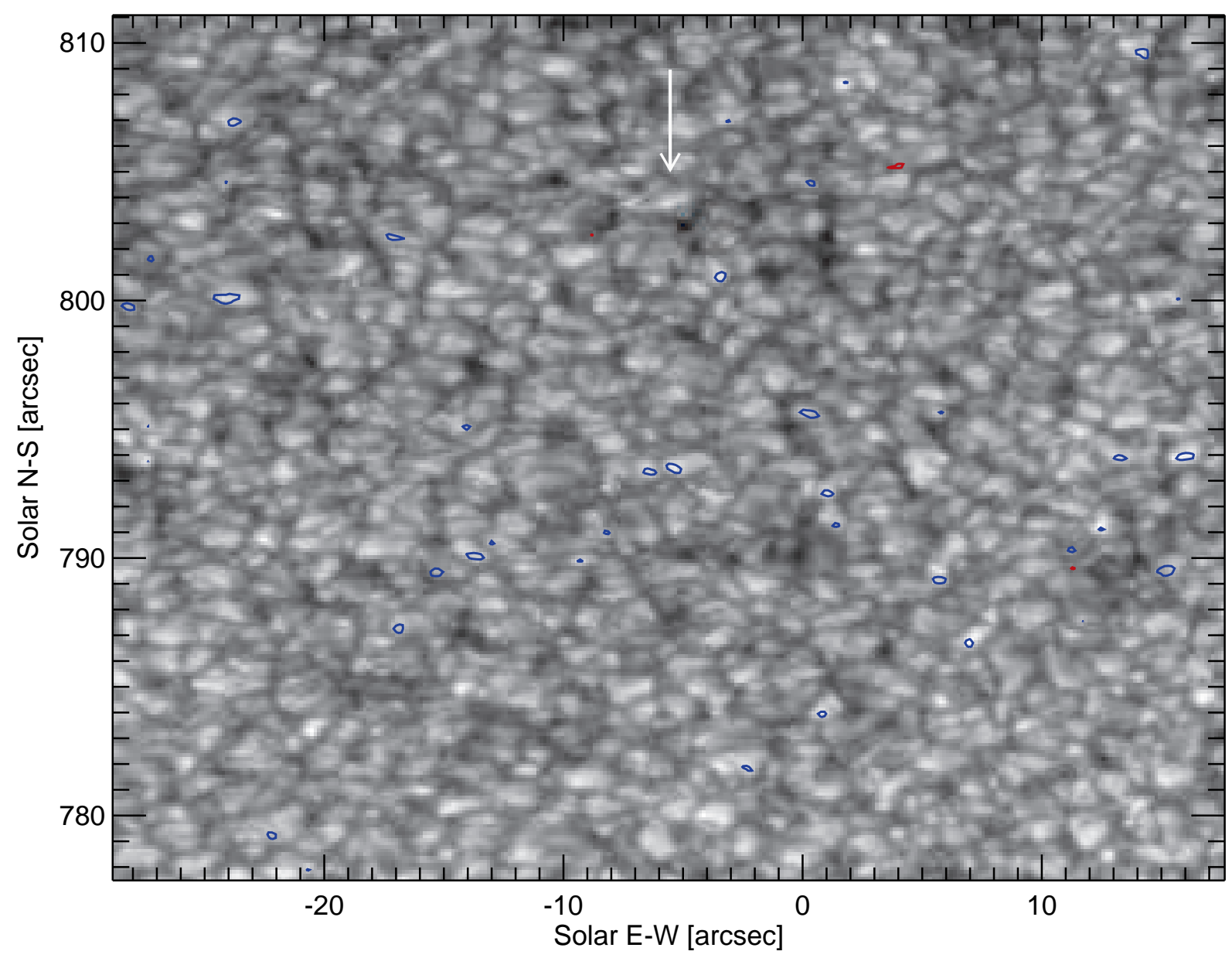

FIG. 3.- Spatial distribution of supersonic horizontal flows in the solar granulation. The displayed region was observed with the Hinode/SP on September 24, 2007 between 08:15 and 09:24 UT. North is up and West to the right. The direction to disk center is indicated by the arrow. Blue and red contours identify locations where the Fe I $630.15 \mathrm{~nm}$ bisectors at the $70 \%$ intensity level are smaller than $-2.6 \mathrm{~km}^{-1}$ and larger than $+2.6 \mathrm{~km}^{-1}$, respectively.

selected but the second would not. This demonstrates that a threshold of $-2.6 \mathrm{~km} \mathrm{~s}^{-1}$ is actually very conservative.

Figure 3 displays the spatial distribution of fast granular flows in a $46^{\prime \prime} \times 34^{\prime \prime}$ subfield observed on September 24 , 2007. The heliocentric angle varies between approximately $54^{\circ}$ to $58^{\circ}$ from bottom to top in the map. Blue and red contours outline positions with bisector velocites smaller than $-2.6 \mathrm{~km} \mathrm{~s}^{-1}$ and larger than $2.6 \mathrm{~km} \mathrm{~s}^{-1}$, respectively. As can be seen, the stronger blueshifts tend to occur on the centerward side of the granules. Large redshifts are very rare and almost exclusively restricted to intergranular lanes.

The fraction of pixels with strongly blueshifted bisectors is $\sim 0.3 \%$ in the two $46^{\prime \prime} \times 164^{\prime \prime}$ regions observed with the Hinode/SP on September 24, 2007 at coordinates $(x, y) \sim$ $\left(-5^{\prime \prime}, 750^{\prime \prime}\right)$. This value differs from the $3-4 \%$ of surface area quoted by Stein \& Nordlund (1998) as being covered by supersonic flows. However, it is important to realize that the two estimates cannot be compared. The reason is that observations are significantly affected by projection effects. For example, observational studies do not detect half of the strong horizontal flows, namely the ones occurring in the limbward side of the granules. Also, the threshold of $\pm 2.6 \mathrm{~km} \mathrm{~s}^{-1}$ used for the bisector shift at 70\% intensity level may have excluded many pixels with supersonic flows, simply because the LOS projection of the velocity vector is smaller than the adopted threshold. This automatically eliminates pixels away from the granular edges (since the flow is not completely horizontal there) and regions close to disk center (because the LOS projection of a horizontal flow is nearly zero in those regions). Thus, the fractional area of $\sim 0.3 \%$ derived from the Hinode observations represents only a lower limit.

\section{STATISTICAL PROPERTIES}

In this Section the line parameters of pixels harboring fast granular flows are compared with those observed in granules. As in the previous Section, fast flows are selected on the basis of their bisector velocities at the 70\% intensity level. Granules are somewhat arbitrarily defined as structures with blueshifted line cores and continuum intensities above 1.02 (in units of the mean continuum intensity at the same heliocentric distance, to avoid any center-to-limb variation).

Figure 4 summarizes the results of this analysis for the two regions observed on September 24, 2007. A total of 1835 pixels with strong horizontal flows have been identified, while the granular sample contains 155430 pixels (out of 645120 pixels in the FOV). Plotted in the figure are histograms of continuum intensities, bisector velocities at $70 \%$ intensity level, total polarization signals, full widths at half maximum (FWHM), equivalent widths (EW), and line-core intensities, for Fe I $630.15 \mathrm{~nm}$.

The first thing to note is that both fast horizontal flows (solid lines) and granules (dashed lines) span a broad range of pa- 

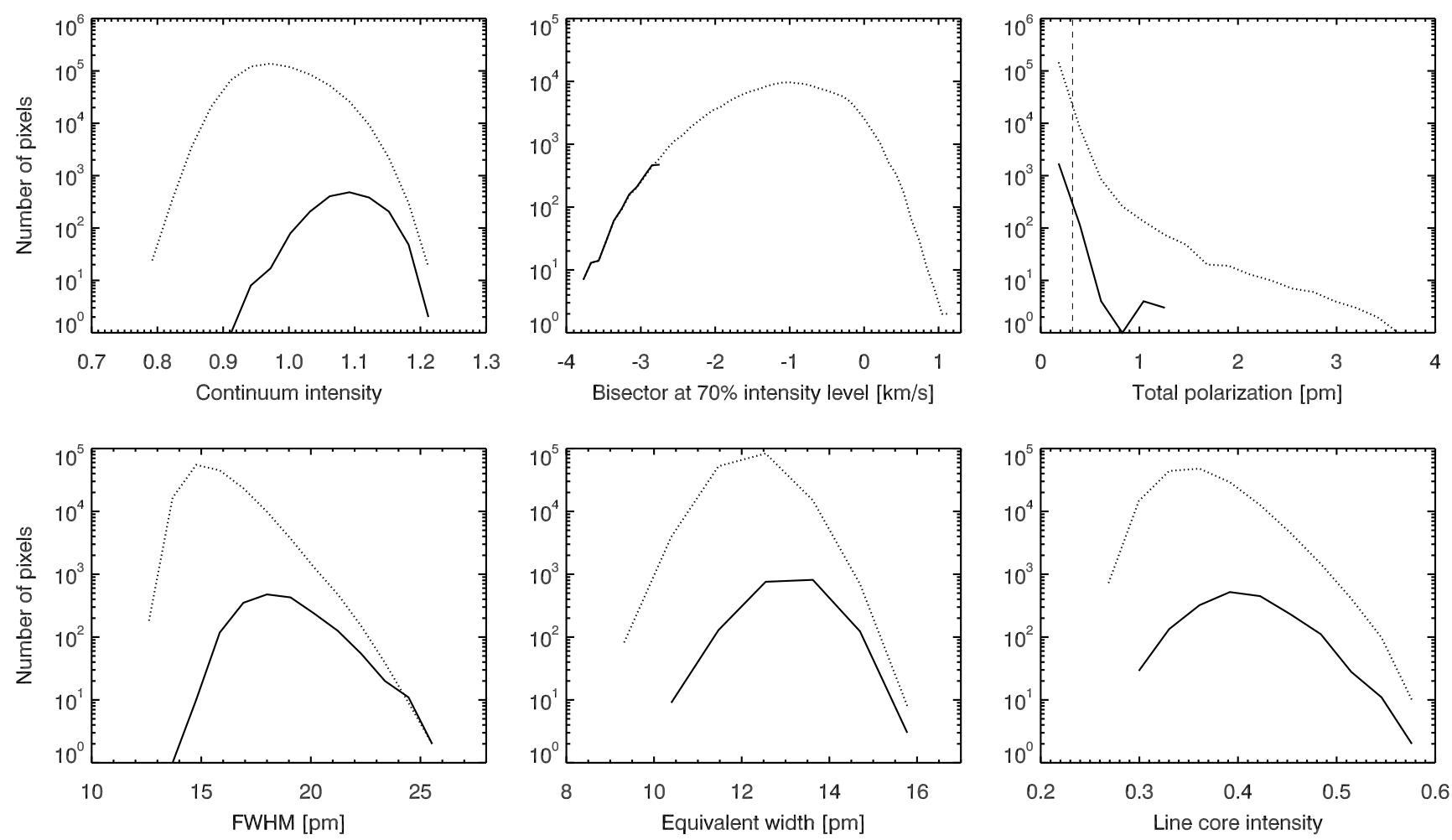

FIG. 4.- Distributions of line parameters for fast horizontal flows (solid) and all granular pixels (dashed) in two scans of a quiet region observed with the Hinode/SP on September 24, 2007 between 08:15 and 09:24 UT, and between 10:10 and 11:19 UT. The region spans a range of heliocentric angles from 45 to $61^{\circ}$, and covers a FOV of $46^{\prime \prime} \times 164^{\prime \prime}$. Top: continuum intensity, bisector velocity at the $70 \%$ intensity level, and total polarization signal. The vertical dashed line represents the estimated noise level in total polarization. Bottom: Full width at half maximum, equivalent width, and line-core intensity. All parameters refer to the Fe I line at $603.15 \mathrm{~nm}$.

rameters. Fast flows tend to be associated with larger values of all the parameters, except for the total polarization signal. However, granules without strong horizontal flows may show similarly large values. This means that detection algorithms based on, e.g., enhanced line widths or equivalent widths will produce many false positives. However, it is also true that the probability of finding supersonic flows increases significantly at the higher end of the FWHM, EW, and continuum intensity distributions. In Figure 4, this can be seen as a gradually smaller vertical distance between the solid and dashed lines as the maximum values of the parameters are approached. An extreme case is provided by the FWHM: all pixels with FWHM values above $\sim 24 \mathrm{pm}$ (approximately 1.5 times the average value in the FOV) are associated with strong horizontal motions. Unfortunately, similar statements cannot be made for the other parameters. Thus, the FWHM is perhaps the best diagnostic of high-speed flows or shocks in granular convection, as first recognized by Solanki et al. (1996).

Figure 4 shows that fast granular motions produce bisector velocities of up to $-3.8 \mathrm{~km} \mathrm{~s}^{-1}$ at the $70 \%$ intensity level, although such extreme Doppler shifts are rare. Another interesting parameter is the total polarization signal. Hinode has discovered that a large fraction of granules harbor magnetic fields (Orozco Suárez et al. 2007). The distributions of Fig. 4 demonstrate that this is also the case in the regions analyzed here. However, supersonic flows are not associated with particularly large polarization signals, as other granular pixels in the FOV show stronger signals (some of them corresponding to granules overlaid by the canopies of magnetic flux tubes in facular and network regions).

\section{TEMPORAL EVOLUTION}

It has been suggested, on the basis of numerical simulations, that supersonic granular motions occur intermittently and exhibit a complex temporal behavior (Cattaneo et al. 1990; Malagoli et al. 1990). These predictions are tested here using time sequences of measurements near the limb.

Figure 5 shows nine raster scans of a small region located $63^{\circ}$ off the disk center. The cadence is 1.9 minutes, a bit long but still adequate to capture the evolution of granular cells. The upper left panel displays continuum intensity maps of the region. In the lower part of the FOV, at $y \sim 1.5^{\prime \prime}$, one can distinguish a relatively large granule that grows, reaches its maximum size, and then starts to decay. During this sequence of events, line satellites can be observed near the granular edge facing the disk center, as illustrated by the spectrograms of Figure $5 b$. Bisector velocities are presented in Figure $5 c$. In this and other panels, contours mark bisector shifts of at least $-2.6 \mathrm{~km} \mathrm{~s}^{-1}$.

During the initial phases of the evolution (first and second scans), no strong velocities are observed anywhere in the granule. Later, as the granule grows in size and brightness, blueshifted satellites appear in its lower half (third to sixth scans). The maximum blueshifts occur in the fifth scan, about 9.6 minutes after the start of the time sequence. There is no smooth variation of the LOS velocity from one map to the next. For example, the bisector shifts are significantly reduced in the fourth scan, to the extent that the strong velocities almost disappear. However, 1.9 minutes later the velocity reaches a maximum. From that point on the blueshifts become smaller. No bisector velocities faster than $-2.6 \mathrm{~km} \mathrm{~s}^{-1}$ 

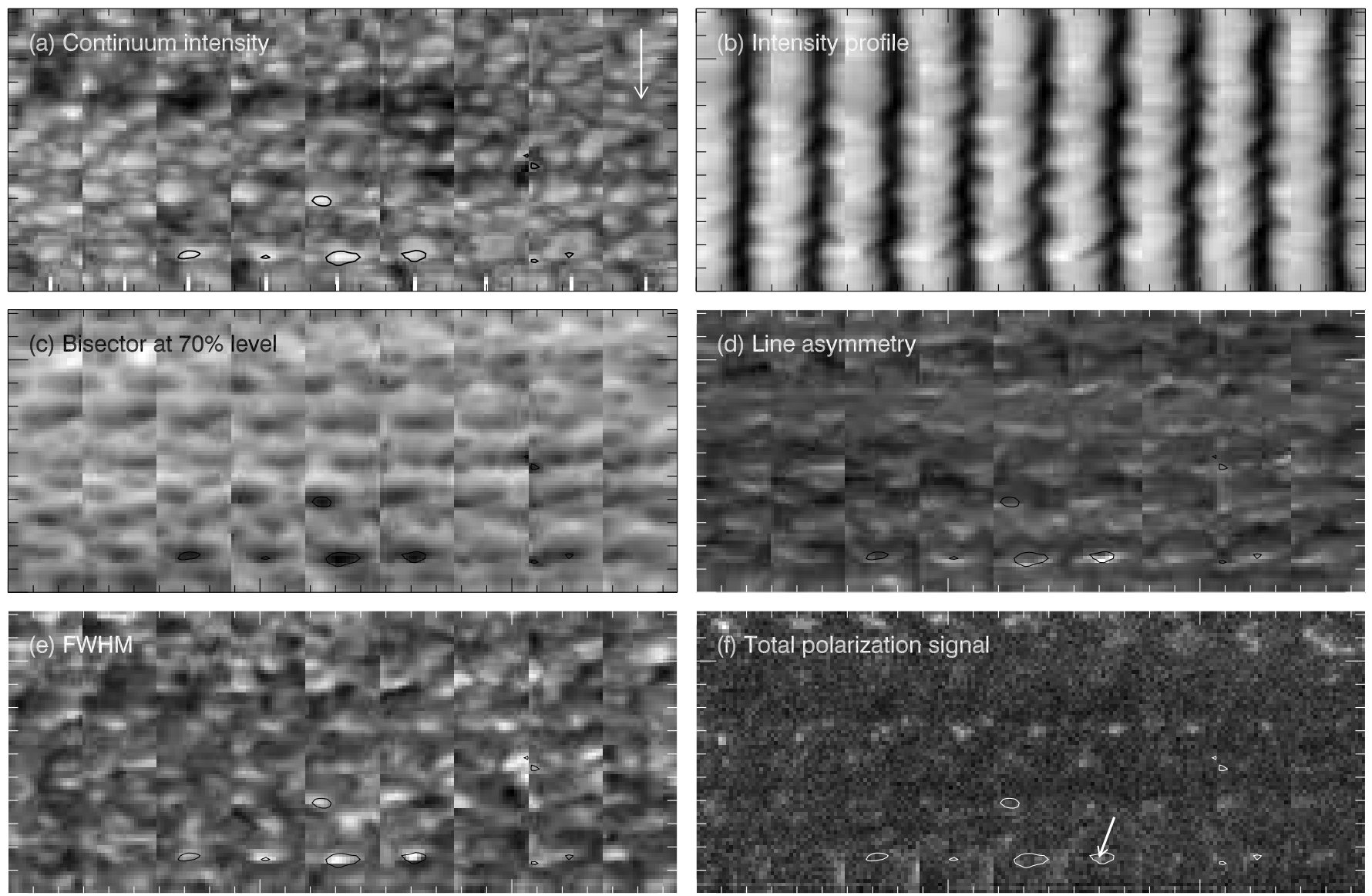

FIG. 5.- Temporal evolution of a $3.0^{\prime \prime} \times 12.2^{\prime \prime}$ quiet Sun area located at a heliocentric angle of $63 \mathrm{deg}(\mu=0.45)$. The observations consist of 9 raster scans made with the Hinode/SP on September 15, 2007, starting at 19:51:05 UT. The cadence is $115 \mathrm{~s}$. North is up and West to the right. The arrow in panel $a$ points to disk center. Tickmarks are separated by $1^{\prime \prime}$. (a) Continuum intensity, scaled between 0.85 and 1.17 of the average quiet Sun value. (b) Fe I 630.15 nm Stokes $I$ profiles along the slits marked with white vertical dashes in panel $a$. The wavelength range goes from -12.4 to $+7.1 \mathrm{~km} \mathrm{~s}^{-1}$. One pixel corresponds to 2.153 pm, or roughly $1 \mathrm{~km} \mathrm{~s}^{-1}$. (c) Bisector position at 70\% intensity level (from -3.7 to $+2.6 \mathrm{~km} \mathrm{~s}^{-1}$ ). (d) Degree of line asymmetry in the wing, measured as the difference of bisector positions at the $50 \%$ and $70 \%$ intensity levels. The scale ranges from -0.7 to $+2.3 \mathrm{~km} \mathrm{~s}^{-1}$. Lage positive values imply lines with very extended blue wings (line satellites). (e) FWHM of the intensity profiles, scaled between $77 \%$ and $137 \%$ of the mean value of 16.2 pm. $(f$ ) Total polarization signal (from 0.22 to $0.55 \mathrm{pm}$ ). The arrow shows the location of the Stokes spectra displayed in Figure 6. In all panels, contours outline structures where the bisector at the $70 \%$ intensity level is blueshifted by more than $2.6 \mathrm{~km} \mathrm{~s}^{-1}$ with respect to the average line core position. They indicate the location of fast horizontal granular flows.

are detected in the seventh scan and only a small patch exhibits strong plasma motions in the eighth scan. After that, the granule decreases in size, fragments, and eventually disappears (not shown). It is remarkable that the maximum velocities occur while the granule is still expanding. Once the granule has grown to its full size, the fast horizontal motions disappear except for the short-lived patch present in the eighth map. All in all, strong flows are observed only during less than half of the granular lifetime.

As can be seen in Figure $5 e$, the largest blueshifts in the FOV are associated with enhanced FWHM values. Both occur inside the granules close to their borders, not in the neighboring intergranular space. This agrees with the findings of Nesis et al. (1993). Intergranular downdrafts are also the site of enhanced line broadening (Nesis et al. 1992; Solanki et al. 1996; Hanslmeier et al. 2008), but their FWHM values are smaller than those detected at the edges of some granules.

The FWHM enhancements may be generated by supersonic flows decaying into shock fronts and producing turbulence (Nesis et al. 1992). An alternative explanation is that the FWHM increases because of large velocity gradients across the shock (Solanki et al. 1996; Rybák et al. 2004). The Hinode data favor the second mechanism, as can be deduced from
Figure $5 d$. Shown there is the evolution of the line asymmetry, defined as the difference of bisector shifts at the 50\% and $70 \%$ intensity levels. Large values of this quantity imply very extended blue wings and/or line satellites, which can be interpreted as being due to strong velocity gradients. In Figure $5 d$, the maximum line asymmetry is reached in the sixth scan, coinciding both in time and space with the maximum of the FWHM.

On the other hand, strong horizontal flows tend to be associated with the brightest parts of granules. In the fifth and sixth scans, the granule near $y \sim 1.5^{\prime \prime}$ undergoes a small brightness enhancement of about $2-3 \%$ at the position of the maximum blueshifts. The same behavior is observed in the granule at $y \sim 4^{\prime \prime}$ (fifth scan). Continuum intensity enhancements at the edges of granules were reported for the first time by de Boer et al. (1992). In numerical simulations, the granular edges are brighter than the centers because of their larger vertical velocities. The transient brightenings observed in Figure 5 may be due to stronger vertical flows or to the development of shocks, which would release energy and heat the plasma. Distinguishing between the two possibilities requires an analysis of the evolution of the granular flow and brightness based on higher cadence observations. 
The two granules of Figure 5 with supersonic flows exhibit weak (but clear) polarization signals toward the end of their lives, as can be seen in panel $f$. An example of the Stokes spectra emerging from the edge of the granule at $y \sim 1.5^{\prime \prime}$ is given in Figure 6. No linear polarization is detected above the noise level, hence Stokes $Q$ and $U$ are not plotted. The circular polarization profiles (Stokes $V$ ) show three lobes, indicating a complex magnetic topology at that position. Very remarkably, the Stokes $V$ lobe placed at shorter wavelengths is strongly blueshifted, just as the line satellite observed in the intensity profile. This suggests that the magnetic fields producing the Stokes $V$ signal are associated with strong horizontal granular motions. One may speculate that these flows are able to drag magnetic field lines and concentrate them at the border of the granule or the adjacent intergranular lane, where they become visible. The field lines would emerge into the photosphere through the granules and may correspond to the small-scale magnetic loops discovered by Centeno et al. (2007) internetwork regions of the quiet Sun. Further observations with higher cadences and lower noise levels are required to confirm or disprove this scenario.

\section{SUMMARY AND CONCLUSIONS}

Hinode observations of the quiet Sun at large heliocentric distances show that double-peaked intensity profiles occur frequently on the centerward side of granules. They consist of a main absorption feature roughly centered at the position of the average profile and a strongly blueshifted, usually weaker, line satellite. Satellites are never seen in the red wing of the intensity profiles or on the limbward side of the granules.

Sometimes the satellites are nearly resolved, in the sense that their cores show up prominently away from the main absorption feature. In those cases, their Doppler shifts indicate supersonic LOS velocities of up to $\sim 9.0 \mathrm{~km} \mathrm{~s}^{-1}$. The sound speed ranges between 7 and $8 \mathrm{~km} \mathrm{~s}^{-1}$ at the formation level of the Fe I $630 \mathrm{~nm}$ lines, hence the Mach number of the granular flow is at least 1.2. Even higher values can be expected in the cooler regions, where the sound speed may reach $6.5 \mathrm{~km} \mathrm{~s}^{-1}$.

At the edge of the granular cell, the satellite exhibits the largest wavelength shift and is usually weak. Toward the granule center, along the line of symmetry, the satellite becomes stronger but its wavelength shift decreases, until it eventually merges with (or replaces) the central absorption feature. This variation of the Doppler shift is consistent with a radial outflow becoming more horizontal as the granular edge is approached. The visibility of the satellites increases toward the limb, due to a more favorable projection of the velocity to the line of sight. However, very close to the limb the satellites disappear, presumably because the line is no longer formed in the layers where overshooting convection occurs. The exact reason can only be found from a detailed analysis of hydrodynamic simulations.

Highly asymmetric granular spectra were known to exist from earlier reports (e.g., Solanki et al. 1996), but this is the first time that satellites are detected unambiguously. The discovery has been made possible by the high spatial resolution, stability, and seeing-free conditions provided by Hinode. The supersonic flows analyzed in this paper share some similarities with those observed by Shimizu et al. (2008) and Nagata et al. (2008) in strong flux concentrations of the network. The fundamental difference is that the latter are the result of physical processes involving magnetic fields, while the former are caused by turbulent convection without the interplay of magnetic fields.
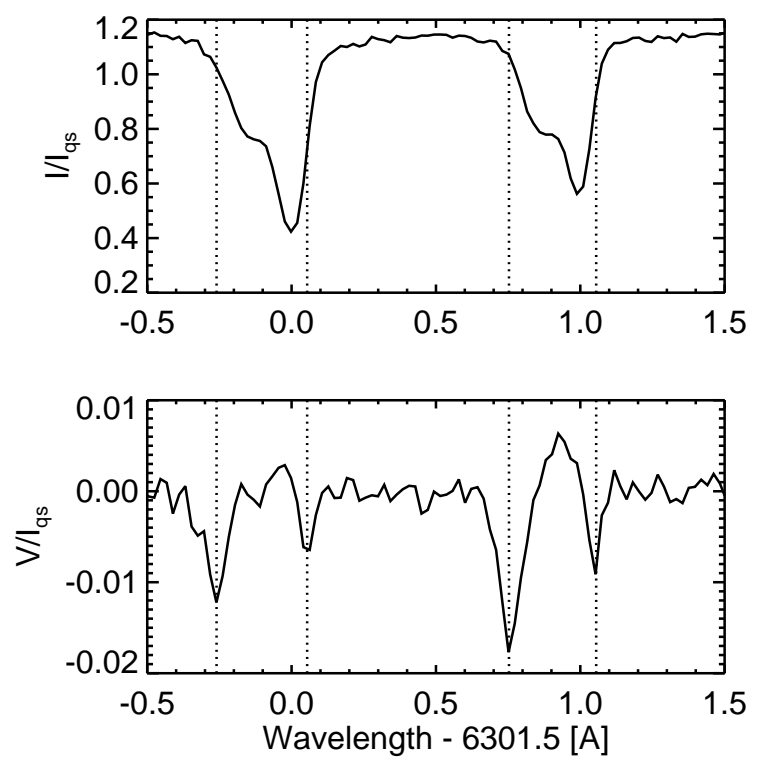

FIG. 6.- Intensity and circular polarization profiles emerging from the edge of a granule harboring strong horizontal flows. The exact location of the profiles is indicated with an arrow in the sixth map of Figure $5 f$. Until that moment, no clear polarization signals were detected in the granule or the adjacent intergranular lane. The zero of the wavelength scale represents the average line core position of Fe I $630.15 \mathrm{~nm}$ over the FOV. The vertical lines mark the wavelength positions of the outer Stokes $V$ lobes.

Supersonic granular flows can be identified through bisector shifts of more than $-2.6 \mathrm{~km} \mathrm{~s}^{-1}$ at the $70 \%$ intensity level. It is important to go close to the continuum to pick out also the weakest satellites. In the Hinode scans, at least $0.3 \%$ of the pixels harbor fast horizontal flows. This value must be regarded as a lower limit due to strong observational limitations. As such, it is consistent with numerical models predicting supersonic flows in 3-4\% of the solar surface at any time (Stein \& Nordlund 1998).

The strongest flows tend to be associated with high continuum intensities, broad profiles, and large equivalent widths. However, the correlation is not perfect and high values of these parameters do not necessarily imply supersonic flows. The Hinode observations suggest that the enhanced line width (quantified in terms of the FWHM) is due to large velocity gradients along the line of sight, not to increased turbulence. The reason is that the largest FWHM values occur within the granule where the line asymmetry (defined as the difference of bisector shifts at the $70 \%$ and $50 \%$ intensity levels) is maximum. Strong velocity gradients can be expected from shock fronts, but they could also be the result of rays crossing intergranular lanes before reaching the granular flow.

As predicted by numerical simulations (e.g., Cattaneo et al. 1990; Malagoli et al. 1990), supersonic granular motions are observed to occur intermittently both in time and in space. First results of an analysis of time sequences near the solar limb suggest that strong flows develop only during half or less of the granule lifetime. However, the cadence of the observations was not optimum and this result needs to be confirmed with additional observations.

Supersonic flows are expected to produce shocks when they brake abruptly at the edge of granular cells. Actually, shock signatures have been observed by Rybák et al. (2004). The data presented here reveals that the strongest flows are associated with small continuum intensity enhancements of only 2$3 \%$. This appears to confirm the predictions of some numeri- 
cal simulations that shocks do not cause significant brightness enhancements (Steffen et al. 1994). The possibility of shocks giving rise to perturbations propagating to chromospheric layers, however, remains to be investigated.

Supersonic flows could also contribute to the emergence of magnetic fields in granules. The gentle granular upflows may take field lines from subsurface layers to the photosphere. Fast horizontal flows would then concentrate the fields at the granular borders and/or the neighboring intergranular space, where they would become visible after reaching a minimum flux density. Support for this scenario is provided by the observation of weak but clear magnetic signals at the edge of granules harboring strong horizontal flows. The Stokes $V$ profiles recorded at those locations are anomalous and often show three lobes. At least one of them is strongly blueshifted, indicating that the magnetic field moves with supersonic speeds. It is tempting to associate this process with the emergence of small-scale magnetic loops in granules (Centeno et al. 2007), but a definite confirmation requires additional observations with better signal-to-noise ratios.

Given their small spatial extent (less than $0.5^{\prime \prime}$ ), a detailed characterization of high-speed granular flows calls for nearlydiffraction limited spectroscopic measurements under seeingfree conditions. At present only Hinode can provide such observations, but hopefully the Imaging Magnetograph eXperiment (IMaX; Martínez Pillet et al. 2004) aboard SUNRISE (Barthol et al. 2006) will be able to take them in the near future. IMaX will not only improve the spatial resolution up to $0.1^{\prime \prime}$ (which is especially important for limb observations), but will also permit faster scans due to the large photon collecting power of its $1 \mathrm{~m}$ telescope.

Hinode is a Japanese mission developed and launched by ISAS/JAXA, with NAOJ as domestic partner and NASA and STFC (UK) as international partners. It is operated by these agencies in co-operation with ESA and NSC (Norway). This work has been partially funded by the Spanish MICIN through projects ESP2006-13030-C06-02 and PCI2006-A70624, and by Junta de Andalucía through project P07-TEP2687.

\section{REFERENCES}

Barthol, P., Gandorfer, A.M., Solanki, S.K., Knölker, M., Martínez Pillet, V., Schmidt, W., Title, A.M., \& the SUNRISE Team 2008, Advances in Space Research, 42, 70

Bellot Rubio, L.R. 2009, in Magnetic Coupling between the Interior and the Atmosphere of the Sun, eds. S.S. Hasan \& R.J. Rutten, Astrophysics and Space Science Proceedings, Springer-Verlag, Heidelberg, Berlin, in press, |arXiv:0903.3619

Bumba, V. 1960, Izv. Crim. Astrophys. Obs., 23, 253

Cabrera Solana, D., Bellot Rubio, L. R., \& del Toro Iniesta, J. C. 2005 A\&A, 439, 687

Cattaneo, F., Hurlburt, N.E., \& Toomre, J. 1990, ApJ, 349, L63

Centeno, R., et al. 2007, ApJ, 666, L137

de Boer, C.R., Kneer, F., \& Nesis, A. 1992, A\&A, 257, L4

Gadun, A. S., Solanki, S. K., \& Johannesson, A. 1999, A\&A, 350, 1018

Gingerich, O., Noyes, R. W., Kalkofen, W., \& Cuny, Y. 1971, Sol. Phys., 18, 347

Hanslmeier, A., Kučera, A., Rybák, J., \& Wöhl, H. 2008, Sol. Phys., 249, 293

Kosugi, T., et al. 2007, Sol. Phys., 243, 3

Lites, B. W., Elmore, D. F., \& Streander, K. V. 2001, ASP Conf. Ser., 236, 33

Malagoli, A., Cattaneo, F., \& Brummell, N. H. 1990, ApJ, 361, L33
Martínez Pillet, V., et al. 2004, Proc. SPIE, 5487, 1152

Nagata, S., et al. 2008, ApJ, 677, L145

Nesis, A., Bogdan, T. J., Cattaneo, F., Hanslmeier, A., Knölker, M., \& Malagoli, A. 1992, ApJ, 399, L99

Nesis, A., Hanslmeier, A., Hammer, R., Komm, R., Mattig, W., \& Staiger, J. 1993, A\&A, 279, 599

Orozco Suárez, D., et al. 2007, ApJ, 670, L61

Ploner, S. R. O., Solanki, S. K., \& Gadun, A. S. 1999, A\&A, 352, 679

Rybák, J., Wöhl, H., Kučera, A., Hanslmeier, A., \& Steiner, O. 2004, A\&A, 420,1141

Shimizu, T., et al. 2008, ApJ, 680, 1467

Solanki, S. K., Rüedi, I., Bianda, M., \& Steffen, M. 1996, A\&A, 308, 623

Steffen, M., \& Freytag, B. 1991, Reviews in Modern Astronomy, 4, 43

Stein, R. F., \& Nordlund, A. 1989, ApJ, 342, L95

Stein, R. F., \& Nordlund, A. 1998, ApJ, 499, 914

Steiner, O., Grossmann-Doerth, U., Knölker, M., \& Schüssler, M. 1998,

ApJ, 495, 468

Tsuneta, S., et al. 2008, Sol. Phys., 249, 167

Wiehr, E. 1995, A\&A, 298, L17 